\title{
Application of Augmented Reality Technology in Chemistry Experiment Teaching
}

\author{
Yan Wang \\ School of Architecture \\ Harbin Institute of Technology \\ Harbin, China
}

\author{
Nan Chen \\ School of Architecture \\ Harbin Institute of Technology \\ Harbin, China
}

\begin{abstract}
Chemistry experiment is an important part of chemistry teaching. Using augmented reality technology to design virtual chemistry experiment is a new way to effectively solve the problem of traditional chemistry experiment teaching. The system takes mobile terminal as its platform, takes mobility and openness, three-dimensional simulation scene, natural interaction and instant feedback as its design principles, and takes Unity3D and augmented reality technology as its development means. Augmented reality virtual chemistry experiment system has three functional modules: learning knowledge, doing experiments and measuring learning effect. The system transforms the traditional chemical experiment teaching into a mobile natural interactive virtual experiment form, which will greatly enhance the students' sense of autonomy and participation, and bring new changes to the chemical experiment teaching.
\end{abstract}

Keywords - augmented reality; virtual chemical experiment; natural interaction; mobile learning

\section{INTRODUCTION}

Chemistry experiment is an important part of chemistry teaching. The experiment process can train students' practical ability, thinking ability, knowledge understanding ability and innovation ability. However, at present, there are many problems in chemical experiment teaching, such as too many demonstration experiments, limited experimental teaching time, space and manpower [1], insufficient experimental equipment, poor maneuverability, potential safety hazards, and test-oriented Experiment-Assisted teaching materials.

\section{APPLICATION BACKGROUND OF AUGMENTED REALITY TECHNOLOGY IN CHEMISTRY EXPERIMENT TEACHING}

Using new technology to design virtual chemistry experiment is a new way to effectively solve the problem of traditional chemistry experiment teaching. For example, the University of Colorado in the United States has designed interactive virtual experiments. The system can provide close to real simulation results, and students can use it to explore learning. In China, Jinhuake Company has designed a simulation laboratory. Chemistry teachers can set up the experimental process and steps according to their needs and students can carry out virtual experiments according to the teachers' settings [2]. Zhang Xuejun used FLASH 3D technology to develop a three-dimensional simulation experiment system, which realized the whole process management of submitting and reviewing the experiment report online, including assistant teaching, independent experiment and experiment report [3]. These virtual experiments have greatly improved the traditional chemical experiment teaching, but at present, the common problems existing in the existing virtual experiment systems are that they are not cross-platform, the interface design is not friendly enough, the interaction form is single, and there is a gap with the real experimental perception.

The maturity of augmented reality technology provides a new solution to the above problems. A technical means of integrating virtual with reality, augmented reality technology has been widely used in educational products in recent years. In English teaching, students scan the English words on the cards, and then they can see the relevant three-dimensional model display, which promotes students' interest in learning [4]. In physics teaching, physical concepts such as electric field and magnetic field are not easy to observe directly. Using augmented reality technology and somatosensory technology, students can visually observe and interact with the magnetic field [5]. In chemistry teaching, there are also some chemicals that cannot be observed directly: such as atoms, molecular structures, etc. Scholars have designed a virtual system of material structure by using augmented reality technology. Students can observe three-dimensional crystal structure more intuitively and naturally [6]. This design idea is also applicable to chemical experiment teaching, that is, using augmented reality technology to display and operate three-dimensional chemical experiment equipment which is inconvenient to use at any time in reality. Augmented reality can provide new technical support for virtual chemistry experiment, provide more simulation experimental equipment, more natural interaction mode, more real experimental experience for students, and bring new changes to chemical experiment teaching.

\section{DESIGN CREATIVITY AND PRINCIPLES OF AugMENTED REALITY VIRTUAL CHEMISTRY EXPERIMENT SYSTEM}

The design of augmented reality virtual chemical experiment system aims to enable students to conduct virtual chemical experiments at any time and place. It can not only 
more accurately simulate the chemical experiment process, so as to obtain more realistic experimental experience. but also conduct experimental operations in the form of natural interaction, so as to obtain the same real chemical experiment experience. At the same time, we should improve the virtual experiment system and add the test module in the experiment system so that students can check the learning results at any time after they complete the experiment operation. This is not only a virtual chemical experiment, but also an interactive experiment teaching aided material which is portable and visually intuitive for students. The design principles of augmented reality virtual experiment are as follows:

\section{A. Mobility and Openness}

The virtual experiment system can be installed in the mobile terminal. Students can learn at anytime and anywhere without the limitation of the time and place of traditional chemical experiment. Another advantage of virtual experiment is that it can share resources with the help of the Internet, update the system anytime, anywhere, and has a strong openness.

\section{B. Three-dimensional Simulation Scene}

Compared with most two-dimensional virtual experiments, the three-dimensional simulation system can

\section{Natural Interaction}

The traditional man-machine interface is more computercentered, and even requires users to think in a computer way. Interaction also tends to be keyboard, mouse, or touch screen. Natural interaction refers to the interaction between human beings and systems in a way more similar to that of real experiments. Students can interact with the system on the basis of their own experimental knowledge and skills, without violating the experimental operation habits and daily behavior, and ultimately complete the experimental tasks simply, clearly and naturally.

\section{Real-time Feedback}

Real-time feedback is a natural process in the real experiment. When students use the virtual chemistry experiment system of augmented reality, they can get instant feedback between the experimental steps. The feedback form includes interface information and voice prompt, which can help students adjust and optimize quickly.

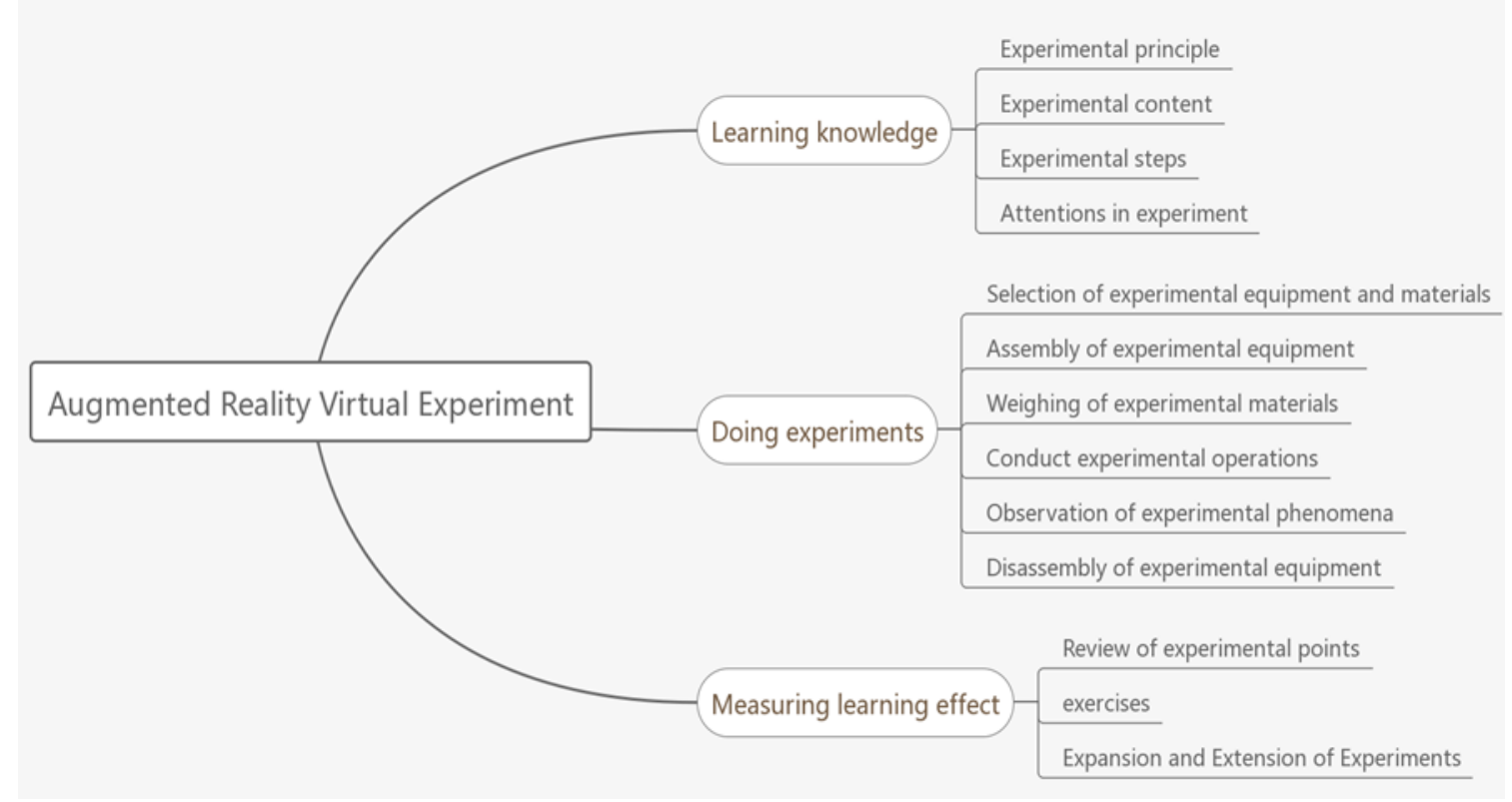

Fig. 1. Structural diagram of augmented reality virtual experiment module.

\section{DESIGN, IMPLEMENTATION AND APPLICATION OF AUGMENTED REALITY VIRTUAL CHEMICAL EXPERIMENT SYSTEM}

\section{A. Module Structure Design of Augmented Reality Virtual Chemical Experiment System}

"Learning Knowledge" module: Students learn experimental knowledge before the experiment, understand the objectives, methods and principles of the experiment, and have a conceptual understanding of the basic knowledge of the experiment. (as can be seen from "Fig. 1")

"Doing experiments" module: Students need to choose the right experimental equipment and materials according to the knowledge module. According to the experimental steps of the system, the assembly of experimental equipment, weighing of experimental materials, experimental operation, observation of experimental phenomena, disassembly of experimental equipment and other operations are completed in turn. (as can be seen from "Fig. 1") 
whether the experimental operation of students is standardized, and give real-time text and voice prompts.

main points of the experimental system, checking exercises, expanding and extending functions, students' ability to learn and use in daily life is improved. (as can be seen from "Fig. $1 ")$

\section{B. Realization Scheme of Augmented Reality Virtual Chemical Experiment System}

In the early stage of system development, first of all, it is necessary to collate the experimental knowledge and design the experimental steps. According to the module structure of virtual chemistry experiment system of augmented reality, in the module of "learning knowledge" and " measuring learning effect ", it is necessary to collate the relevant pictures, texts, audio and video data of each experiment for subsequent development and display. In the "doing experiments" module, we need to sort out the equipment and materials needed for the experiment, and design the experimental steps to clarify the objectives, operation methods and operation results of each step. For example, if the goal of a step is to absorb a solution, then the operation mode is to move the three-dimensional virtual model of the rubber head dropper and insert it into the solution bottle. The operation result is that the head of the rubber head dropper absorbs the solution.

The modules of "learning knowledge" and "measuring learning effect" mainly use Unity 3D software to realize the virtual chemical experiment system based on mobile terminal. The system uses $\mathrm{C} \#$ language to develop the interface at all levels, and introduces and tests the experimental knowledge. Mobile terminals need to present complex content in a smaller interface. Therefore, in the design of digital content, we should display complex information with graphics, audio and video as far as possible. The presentation of information should be hierarchical and easy for students to understand.

The "doing experiment" module is the core of the system. In the early stage, the materials and steps required for the experiment have been clearly defined. This part develops and realizes the experimental process. This system uses Vuforia SDK to realize the augmented reality function. By scanning the two-dimensional recognition map with the mobile camera, we can see the three-dimensional virtual experimental equipment from the mobile screen. Firstly, two-dimensional identification maps of each piece of experimental equipment need to be made import them into Qualcomm Vuforia official website and print them as cards for reserve. Then we need to use MAYA to model the experimental equipment, import the three-dimensional model into Unity3D, and import Qualcomm's Vuforia SDK. The function that needs to be developed is to display the threedimensional virtual experimental equipment model on the recognition card when the recognition map is detected. The students move the recognition map by hand, and the virtual experimental equipment will move along with it. According to the specific requirements of the experimental steps, the system needs to acquire the position of the equipment in real time, use the collision detection system of Unity 3D to judge

\section{Application Example of Augmented Reality Virtual Chemical Experiment System}

The first module of Augmented Reality Virtual Chemistry Experiment System is to learn experimental knowledge. Students can choose experiments they are interested in, and then study the experimental principles, contents, steps and precautions.

After having a basic understanding of the experiment, students can enter the experimental module. Students need to choose experimental equipment according to their understanding of the experiment, and identify the experimental equipment card under the mobile camera. After the system recognizes correctly, it will display the threedimensional model of the equipment and introduce the information of the equipment by voice. The experiment can be started after the students correctly select all the equipment needed for the experiment. In the experiment, each step will have corresponding prompts, students will move the recognition card, and the three-dimensional model of the experimental equipment will move along with it. Therefore, students can move the experimental equipment naturally with their hands, just like real experiments. Then through the interaction between the experimental equipment, each step of the experiment is carried out in turn. According to the specific requirements of the experimental steps, the system will judge whether the experimental operation conforms to the specifications, and give students real-time feedback in the form of text, voice prompts, etc.

After the completion of the experiment module, students can enter the test effect module, which includes the summary and review of the experimental points, the test of experimental exercises and the analysis of the results, and the expansion and extension of the experiment. In the part of Exercise, students need to recall the experiment process and complete relevant exercises. The system will analyze and give guidance to the students' answers. So far, the students have completed a complete virtual chemistry experiment of augmented reality.

\section{CONCLUSION}

Chemistry experiment teaching is a highly professional field. Traditional experiments require well-equipped laboratories and special instructors. Some dangerous experimental requirements need professional protective facilities. The virtual experiment software of augmented reality based on mobile terminal can give full play to students' subjective initiative to a large extent, and is not limited by time, place, experimental equipment and other restrictions. Teachers can real-time detect students' experimental status through the virtual experiment software, and prompt and guide students in time. At the same time, because it is a virtual experiment, students can move threedimensional experimental equipment in the virtual environment, boldly and carefully carry out dangerous experiments without worrying about the occurrence of health 
risks. Most importantly, augmented reality technology enables students to simulate chemical experiments in a natural way. Of course, one of the problems of augmented reality virtual experiment is that users always need a handheld mobile phone. In the future, virtual chemistry experiment can also use wearable devices, such as direct observation from AR glasses, so users can use both hands to experiment. Intelligent voice interaction technology can also be used for software interface switching, so that the interactive experience of virtual experiment will be more natural.

To sum up, augmented reality virtual chemistry experiment pays attention to students' enthusiasm and sense of participation. It enables students to repeat and complete a closed loop of chemical experiment learning many times in a novel interactive way, which is conducive to improving the learning and teaching effect of chemical experiment. It is a useful exploration of mobile learning mode.

\section{REFERENCES}

[1] Wei Dejing. Problems and Solutions in Senior High School Chemistry Experiment Teaching under the New Curriculum Standard [J]. China Extracurricular Education, 2013 (8): 80. (in Chinese)

[2] Wang Weiguo, Hu Jinhong, Liu Hong. Current situation and development of virtual simulation experiment teaching in foreign universities [J]. Laboratory research and exploration, 2015 (5): 216219. (in Chinese)

[3] Zhang Xuejun, Tang Jiuli, Wei Jiangming. Design and implementation of a virtual experiment platform for middle school chemistry based on Flash3D [J]. Research on audio-visual education, 2014 (1): 79-84. (in Chinese)

[4] He J , Ren J, Zhu G, et al. Mobile-Based AR Application Helps to Promote EFL Children's Vocabulary Study [C]// IEEE International Conference on Advanced Learning Technologies. IEEE, 2014.

[5] Cai S, Chiang F K, Wang X. Using the augmented reality 3D technique for a convex imaging experiment in a physics course [J]. International Journal of Engineering Education, 2013, 29(4):856-865.

[6] Wang Cunyou, Cheng Tong. Overview of the Research on Augmented Reality Educational Applied Products [J]. Modern Educational Technology, 2016, 26 (5): 95-101. (in Chinese) 